\title{
How do GP registrars feel about dealing with mental health issues in the primary care setting? A qualitative investigation
}

Helen Lucas, Amy Scammell and Carolin Hagelskamp Battersea Research Group, Bolingbroke Hospital, London, UK

\begin{abstract}
The treatment of mental health problems is now a national priority in the UK. With a prevalence rate of $23 \%$ in the general population and $75 \%$ of the population visiting a GP each year, the role of primary care in the detection, management and treatment of mental health problems is pivotal. However, around $20 \%$ of mental health problems remain unidentified by GPs. Many reasons may contribute to this. One reason is cited as being the training received by GPs, although research into this has yielded inconclusive results. This study aimed to investigate qualitatively the attitudes of GP registrars, firstly towards dealing with mental health problems in primary care and secondly in relation to the training and support received. Sixteen semistructured interviews were conducted and analysed thematically. Results indicated that GP registrars in this study understood the psychosocial nature of mental health problems and felt confident in detecting these issues in their patients. However, they were less secure about the management of mental health problems due to a number of constraints such as inadequate time, lack of knowledge of referral pathways and local resources, and a limited understanding of the psychology of mental health problems. They appreciated the support provided by their trainers, the training practices and the vocational training scheme (VTS). They would benefit from additional input concerning assessment and management of psychological problems, and discussions around their new role as a GP, acknowledging its potentials and limitations.
\end{abstract}

Key words: detection; GP registrars; management; mental health; support; training

\section{Introduction}

In 1999, Saving Lives: Our Healthier Nation (Department of Health, 1999a) identified mental health problems as a national priority. Two targets were set; first to reduce the suicide rate of 4000 per year by $20 \%$ and secondly to increase the rates of diagnosis and treatment of mental health problems. The National Service Framework for Mental Health (Department of Helath, 1999b) highlighted primary care as a key area in the improvement of mental health care. This focus and effort is well justified. Results from the Gen-

Address for correspondence: Dr Helen Lucas, Battersea Research Group, Bolingbroke Hospital, Wakehurst Road, London, SW116HN, UK. Email: helen.lucas@kcl.ac.uk eral Household Survey (Department of Health, 1995 ) indicated a $23 \%$ prevalence of psychological symptoms in the general population. AyusoMateos et al. (2001) found that urban Ireland and urban UK had the highest prevalence of depressive disorder in Europe, and The World Health Report (World Health Organization 1999) estimated that by 2020 depression would constitute the second most burdensome health problem after coronary heart disease. These estimates reflect not only a real increase in mental health problems, but also a change in the way the medical professions as well as the general public understand and deal with emotional problems.

Ninety per cent of people with mental health problems are treated in primary care (Goldberg and Huxley, 1992). Seventy-five per cent of the population visit their GP each year. Research, 
however, suggests that primary care is not yet adequately equipped to deal with these numbers. There is still serious underperformance in the detection and management of mental health problems in primary care, particularly in terms of depression (Goldberg and Huxley, 1992; Kessler et al., 1999; 2002; Ohayon et al., 1999). It is estimated that half the patients presenting with depressive symptoms are missed on their first consultation. While some people's symptoms improve over time and others are recognized on subsequent consultations, 20\% remain undiagnosed (Kessler et al., 2002; Paykel and Priest, 1992). The picture is further complicated by research that shows detection does not guarantee successful treatment and is therefore not related to outcome (Dowrick and Buchan, 1995). It could be argued therefore that diagnosis by GPs is not the crucial issue. But considering that effective treatments such as medication and counselling are available through the NHS, a GP's diagnosis, when followed by adequate management constitutes a decisive step on the pathway to better health.

Unsurprisingly, the last decade has seen much debate and research into the effectiveness of psychiatry placements for GPs (undertaken by $40 \%$ of British GPs, Ratcliffe et al., 1999) and other community-based training and awareness initiatives targeted at primary care (Gask et al., 1989; Paykel et al., 1997; 1998; Payne et al., 2002; Ratcliffe et al., 1999). However, Hodges et al. (2001) conclude that psychiatry placements fail to meet the needs of GPs and that there are shortcomings in teaching methods. For example, the psychiatric assessment scales and lengthy clinical interviews used in hospital psychiatry do not translate easily to 10 minute GP appointments (Hickie, 1999). Also, the types of mental health problems treated in hospital are largely different from those seen in the community. These discrepancies lead to attitudinal problems in the way GPs and psychiatrists perceive each other, which in turn hinders the learning process. The effectiveness of less formal, primary care-specific training has also been questioned by evaluation. Whilst professionals appreciate additional training, the long-term effects have been difficult to quantify (Kerwick et al., 1997; Rix et al., 1999; Upton et al., 1999; Williams, 1998). This, however, should not undermine the importance of training, but rather emphasizes the social complexity underlying the detection and management of mental health problems in primary care. Other factors need to be taken into consideration, and possibly addressed in training schemes.

Dowrick et al. (2000) and Thompson et al. (2000) found GPs' assessment of their ability to detect depression was unrelated to their actual detection rates. However, their self-assessed level of confidence in relation to dealing with mental health problems and the degree to which they believed that treatment leads to improvement did correlate with their performance. The authors therefore concluded that confidence and optimism are related to detecting and dealing with mental health issues. Cape (2000) has shown that it is not simply the content of the consultation, but also the way the patient perceives its social and emotional dynamics which relate to a reduction of symptom severity. Doctors' communication skills are therefore vital in both the detection and treatment of mental health problems. This is an important finding as some patients' attribution styles make it easier to detect their mental health problems than others. GPs need the skills to distinguish between, as well as relate to, the somatizing, normalizing and psychologizing styles with which patients may present (Kessler et al., 1999), while remaining both task-orientated and effective (Cape, 2000). Finally, factors on the practice level such as a poor cohesive organizational culture, inadequate resources, lower rates of mental health worker allocation and a high deprivation score have been associated with underdetection of mental health problems and highest rates of psychiatric referral (Ashworth et al., 2002; Scott et al., 2002). However, this area needs further exploration.

As Paykel and Priest (1992) describe, GPs face a difficult task in dealing with their patients' mental health issues and are constrained by their work conditions. This is recognized in academic literature, medical education and throughout governmental and voluntary sector publications and initiatives. Newcomers to general practice, such as GP registrars, are therefore likely to be aware of the problem. But how do they feel about the task ahead of them? Are they prepared to detect and manage the growing numbers of patients with depression and anxiety? What are they confident about and where do they think 
they need more support? This paper reports on a study that set out to explore these questions with GP registrars in south west London. Pilot work had shown that GP registrars constitute a distinctively interesting group in this type of research. As doctors still acculturating to general practice as a new working environment, they are in the process of formulating their views and making sense of their everyday experience in relation to their previous expectations, theoretical backgrounds and their new reality.

\section{Aim}

The aim of this paper is to explore GP registrars' experience, attitudes and beliefs about primary care mental health, previous training and current support.

\section{Sample and methodology}

Semistructured interviews were conducted with 16 GP registrars from south west London, all undertaking their vocational training scheme (VTS) at St George's Hospital Medical School. All 25 registrars undertaking the VTS in January 2002 were contacted first by letter. Some initiated contact from this; the others were followed up by telephone. Interviews with registrars that consented to participate were arranged for a mutually convenient time and conducted at their training practice. Participants constituted a convenient, self-selected sample of seven males and nine females, of whom four males and three females had completed a 6-month senior house officer (SHO) job in psychiatry. Interviews were conducted by $\mathrm{CH}$ or $\mathrm{HL}$ and followed the same topic guide covering: mental health training; experiences with detecting and managing mental health problems in primary care; areas of confidence or concern; beliefs about the aetiology of common mental health problems and their treatment; and knowledge of resources and support. All interviews were tape recorded, transcribed and converted into Atlas/Ti, a software package for qualitative analysis that facilitates the identification of themes and patterns of thought. The three authors analysed the data independently according to similarities and differences in emerging themes (Glaser and Strauss, 1967). Analyses were validated against each other and a common coding frame established. All three authors analysed the data a second time, and came together to synthesize the interpretation. Differences in readings mostly reflected the varied backgrounds of the researchers (GP, social psychologist and methodologist). These were resolved by discussion.

\section{Results/Discussion}

To make sense of their ways of dealing with patients' mental health problems, GP registrars drew upon their understandings of themselves as GPs, the nature of general practice, and their beliefs about the aetiology and course of common mental health problems. Distinctions were made between detecting mental health problems and managing them. Confidence was gained through the support of in-practice trainers and other GPs, the VTS and previous training. Sources of insecurity were the conflicting demands of the role of a GP and inadequate knowledge of the psychology of mental health problems, treatment options and local service provision (see Figure 1 for summary).

\section{Thinking beyond the medical paradigm}

All registrars commented on a change in thought that the move from hospital medicine into general practice required. They started to see patients as people with interrelated and often illdefined problems and began to interpret these as being the product of medical, social and psychological factors. As such they had also begun to perceive their roles as GPs and the demands of their professional practice expanding beyond pure medicine.

In hospital you can see them as they've got this symptom, like chest pain etc, but you never actually see them in the outside world [...] they may well be dealing with other ill relatives at home, they may be issues with their jobs etc. As GPs we can see all that. We do not just work as medical practitioners but also as social workers and friends (1). 


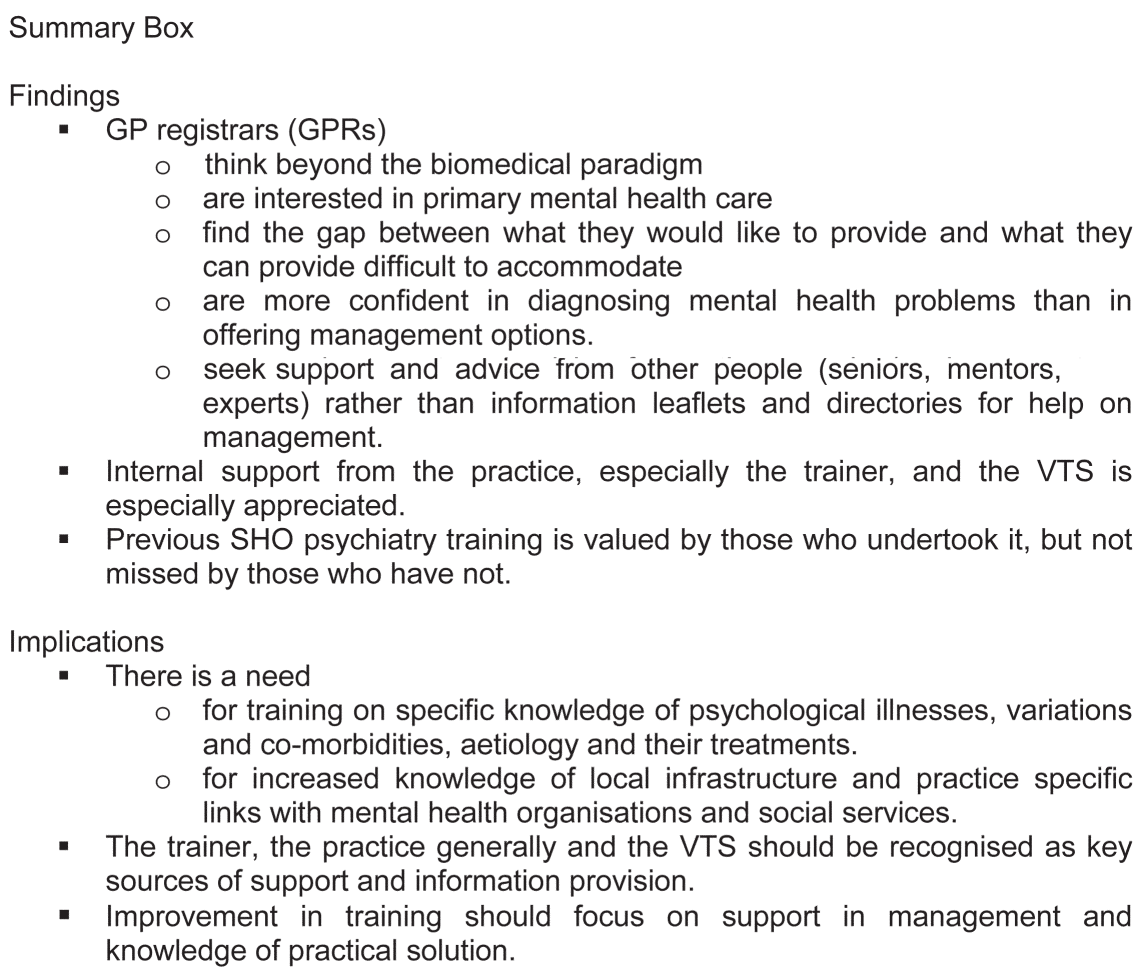

- Internal support from the practice, especially the trainer, and the VTS is especially appreciated.

- Previous SHO psychiatry training is valued by those who undertook it, but not missed by those who have not.

Implications

- There is a need

- for training on specific knowledge of psychological illnesses, variations and co-morbidities, aetiology and their treatments.

- for increased knowledge of local infrastructure and practice specific links with mental health organisations and social services.

- The trainer, the practice generally and the VTS should be recognised as key sources of support and information provision.

- Improvement in training should focus on support in management and knowledge of practical solution.

Figure 1 Summary of results

A GP is what the vicar used to be. You are there for everyone and anything (3).

I think a lot of GPs would say that it is like solving a puzzle every ten minutes. And a puzzle that is incredibly complex and it involves a human being (14).

Most evaluated this shift in thought and practices positively. They felt rewarded by the enhanced doctor-patient relationship and enjoyed the challenges posed by the variety and ambiguity of problems with which their patients presented. Registrars did differ though in the pace by which they became comfortable in the new environment. Some were more wary about the holistic role of the GP and the need to deal with a range of problems in one's patients. Specifically, these registrars identified the uncertainty of diagnoses as challenging. However, this was not seen as insurmountable but rather as an area for further development.

I prefer subjects where there's a kind of definite answer. [General Practice is] all just a bit kind of wishy-washy and you never know whether you've done the right thing (12).

Some registrars have said that they enjoy getting problems that don't strictly fall within medical diagnosis and management. Sometimes I find that a little bit troubling but [...] it is a quality I've got to develop (7).

With other registrars these challenges were also a source of frustration. On occasions when they felt unable to provide answers, some felt inadequate, as if they were failing themselves and their patients. Others felt under pressure and that their 
medical legitimacy was scrutinized by patients due to their registrar status.

Often people have sort of behavioural difficulties like blushing in public or people say they are easily embarrassed or get very nervous. I feel silly making suggestions that probably make it worse (4).

Patients [are] not confident about what you're doing which is difficult as a registrar [...] some of them challenge what you're suggesting is right and [...] often they'll come in with things I don't know about and they've got off the internet (14).

These accounts demonstrate how GP registrars are still working to establish boundaries to their roles and specific identities. These need to be achieved to allow themselves to gain satisfaction from their work, judge their impact and become comfortable with their limitations. Given the importance of these issues on a practical level and the emphasis government policies place on multidisciplinary health and social care, it seems reasonable to suggest that GP trainers should facilitate constructive discussions about the paradigm shift that needs to be made from hospital medicine to general practice. Explorations of registrars' expectations of their roles and how these may differ from colleagues and patients may be valuable.

\section{Making sense of common mental health problems}

A general consensus prevailed amongst the registrars that mental health problems featured prominently in GP consultations, with depression and anxiety being most common and psychosis being rarely seen. However, when asked to describe how frequently they saw mental health problems, the registrars' accounts varied. While some seemed to think inclusively about psychosomatic presentations as mental health problems, others distinguished those more ambiguous cases from patients who present with classic symptomatology.

I seem to see it all the time. It is amazing how much there is of it. Also, patients who come in for other things, it often says past depression in their notes (2).
I do not see them that much really. I would guess that I see one new depression, new in the sense that I newly recognize and diagnose it about every 5 or 6 weeks only [...] What you see a lot are somatic problems, for which you know it can or is likely to be a depression, that is psychosomatic, or somatizing (3).

I don't actually get many people with psychiatric problems, for some reason. In the 11 months I've been here I've only had a handful which is probably not representative [...] the thing is that many of them present with other problems (16).

These accounts clearly reveal diverse understandings of what constitutes a mental health problem. We asked all GP registrars to what they attributed the psychological difficulties they saw in their patients. Most described relatively broad models of psychosocial causes, emphasizing social problems, environmental factors and life events such as divorce, unemployment, housing problems, as well as concomitant physical illness as the main causes for low mood. Some related depression more generally to uncertainty, loss of identity, greed or boredom.

We see so much depression. Living in London, there is a lot of pressure on people, especially in the deprived areas, but also you get all these women with millionaire husbands who are really unhappy and feel they do not have enough (1).

Mental health problems more so than anything are very much in the context of job, life, relationships, social interactions, accommodation, etc. (9).

This psychosocial model of understanding mental health problems was consistent with the registrars' overall understanding of general practice and their role as GPs as described above. However, it also led one registrar in particular to feel overwhelmed at the potential need for support for mental health problems and to question the effectiveness of psychological or medical treatment. 
I think about a third of the population is probably pretty low at any one time and what do you do with that, how can you what can you do to help and how can you possibly encompass that? (4).

You've got medication, you've got counselling, you've got to refer them, but often none of those three are quite right for them and it's just a matter of being there and listening, but sometimes it doesn't feel as if you're doing enough (4).

Potentially GP registrars could benefit from more detailed knowledge about mental health problems. Training in psychological concepts and aetiological models could help doctors to distinguish between different types of emotional and cognitive states and patients' own attribution styles. As such, the increasing body of mental health promotion literature from both governmental and non-governmental agencies (e.g. Department of Health, 2001; Mind, 1998; The European Network for Health Promoting Agencies, 2003) and current research into somatization (e.g. Best et al., 2002; Schilte et al., 2001) may help to meet their needs.

\section{Detecting mental health problems}

Despite inherent ambiguities, most registrars reported feeling confident about their skills in detecting depression. Many expressed a specific interest in the field of mental health, finding it a rewarding area, and few were hesitant about asking sensitive questions, for example, regarding suicidal ideation. Most preferred clinical interview techniques, asking patients about symptoms defined in the Diagnostic and Statistical Manual of Mental Disorders IV (DSM) (The American Psychiatric Association, 1994), rather than administering standardized assessment scales such as the general health questionnaire (Goldberg and Williams, 1991). Thus, at least in their own eyes, detection and diagnosis of mental health problems did not seem to pose a difficulty.

I feel confident about dealing with situations where the patient is quite low but is not necessarily recognizing that (4).

I feel really confident doing it. I am not saying I am a great psychiatrist, rather I feel that I am really not afraid at all to talk to patients about problems that are quite intimate or personal, things you normally do not talk about (3).

It's [suicidal ideation] one of the questions that you must ask. Except that you generally don't come out straight away with saying 'Are you suicidal?' we're generally taught to approach it in a slightly more gentle, oblique way (7).

However, personally detecting mental health problems did not necessarily translate into anything further. Registrars reported that they did not always discuss their concerns with the patient or manage these immediately. Dealing with depression and the subsequent disentangling of associated issues is time consuming. Consequently 'lack of time' was the main reason cited as to why they might decide not to fully explore a mental health issue during the first consultation. The pressure to prioritize - giving one patient more space to talk than another or deliberately ignoring a hidden agenda - was seen as inevitable with 10 minute appointments.

Sometimes you are running so late and you are aware that there may be other agendas and you just have got no time to get into them (12).

Some registrars justified these actions through arguments relating to the organization and ethos of primary care:

Doctors have a responsibility for their patients not to run too late (12).

The point about general practice medicine is that it is ongoing and there's always the chance to come back and the patients know it so even if they don't quite get up the nerve to talk about something on one occasion they probably will be back again quite soon and probably feeling more able to talk about things the next time round (7).

However, these justifications are problematic. As previously stated, it is too optimistic to believe that all depressions are eventually diagnosed. 
Also, postponing a diagnosis runs counter to the objectives of the National Service Framework for Mental Health (Department of Health, 1999) which advocates early intervention and fast and effective treatment to reduce the risk of wider effects on a person's physical health and social environment. Some effort may be well used to investigate how detection and diagnosis of mental health problems can be made more effective, while considering resource constraints and accounts that suggest that diagnosing mental health problems constitutes a process rather than a single act. As a process it does require some degree of negotiation and compromise spread over several consultations, in order to engage the patient's trust and ultimately benefit the patient. Future research should look at this process more carefully to identify the beneficial and less beneficial elements.

\section{Managing mental health problems}

Ideally, systematic evidence-based management immediately follows a diagnosis. However, registrars tended to speak about detection and management of mental health problems separately. For most, managing mental health problems in primary care constituted an area of substantial uncertainty. Again, insecurities about patients without clear-cut diagnoses are recognizable here.

I find the follow-up consultations really much more difficult. Once you have actually told someone 'you are feeling low' and gone through the 'whys' - I mean where do you go from there? (4).

You have patients coming in who say they are miserable and you do the depression screen and they have the biological symptoms and you think 'this is great', you've got treatment. And then you get people coming in saying the same things and they don't necessarily have the symptoms and they're the people you don't really what to do with (9).

Many registrars said they took management upon themselves in terms of following up particular patients.
We can see them on a regular basis. That helps people over a crisis type of thing. If I [saw] them possibly last Monday, I may see them again on Friday and then I will see them again on Wednesday. You know, just to see how they are getting on (1).

By the nature of their positions, none had any experience with long-term treatment and therefore had not yet been able to see benefits of some treatments over others. This may explain why many were still relatively insecure about mental health management and doubted the extent to which what they could offer would actually make the difference to the misery they were seeing.

Many people need a reasonably good duration of counselling and here they get referred and they may or may not have counselling and if they do they get six sessions. What you can achieve in six sessions I'm not really sure when you've got issues of self-esteem and stuff which are impacting quite majorly on how you function in the world (1).

Unlike other chronic diseases, there is little useful hard data to audit within mental health and primary care, which, again, makes measuring performance by outcome difficult. Therefore, we discussed with the registrars to what extent knowledge of local resources, previous training and current support facilitated confidence in the management of mental health problems.

\section{Knowledge of resources}

In terms of effective management of mental health problems, knowledge of local resources available to practitioners and patients is vital. Some registrars had clearer ideas about local referral pathways than others. As such some were very resourceful and imaginative in their approaches to finding appropriate treatments, whereas some only considered medication or in-house counselling. While practice counsellors were commonly attached to the practices, few registrars seemed to be sure of the precise referral criteria and the type of counselling offered. Similarly, although community care teams operated for mental health, these were viewed by some as being inaccessible. 
We only have a private therapist, which I do not know very much about or what they do apart from relationship difficulties, etc (4).

We do not really work in a team. It is quite difficult to get support for individual patients because you are the only one seeing them [...] it is the sort of thing you get used to, but sometimes you feel you would like more support. I guess there is the community mental health team to get in touch with, but it is quite a process to get hold of them (8).

Alternative treatment options and voluntary sector groups were rarely mentioned. Most had received a list of NHS, voluntary and private sector services and support groups with contact details and referral criteria at the beginning of their registrar year, but few seemed to use this regularly. It had either been misplaced or filed away.

There are the volunteer services, I suppose most people find their way to them somehow (2).

When some registrars talked about the local resources a further complicating issue that arose was the oversubscription of many services (for example, in-house counselling can have waiting times of up to three months). In some cases registrars had good knowledge and provided their patients with a good deal of information, but in the end had to suggest that they approached services outside the NHS for appropriate support.

I encourage the patient to approach voluntary sector services directly. Whatever I can find that might suit them. But they do not need me to refer them. I try to make sure they have the information, and you need to explain it to them as well [...] There are also clinical psychologists, but they are very overbooked, so the pastoral services tend to be used more (7).

Local knowledge of appropriate health and social care partners, agencies and services is vital as a GP. It enables the provision of patient-centred and flexible care - something the UK is strategically working towards under the NHS Plan (Secretary of State for Health, 2000). Additionally, being able to discuss a range of different treatment options with patients is likely to increase a registrar's confidence. Considering that most registrars enter a training practice in areas with which they are not previously familiar, it is the responsibility of the training practice to introduce them to their specific referral structures and links, and to encourage them to test and broaden these.

\section{Previous training}

Of the seven registrars that had previously undertaken a psychiatry SHO post, none regretted having done so and all cited benefits including increased awareness of mental states and confidence in dealing with patients' mental health problems.

I think the job in psychiatry did give me confidence, really dealing with the issue and confronting it (15).

It helped me deal with you know the use of medication and what services are offered. I mean I worked in a community-based one, so it was partly in hospital and partly in the community. If I had not done psychiatry I would not know what you know who is out there fiddling between doctors in the hospital and GPs, psychiatric nurses, social workers, how they all interact as a community mental health team, how you can access the system. This takes a long time to pick up in primary care (1).

Due to the differences between hospital psychiatry and primary care mental health, knowledge and communication skills specifically relevant to primary care on the whole had to be acquired in general practice itself and through additional educational support during the registrar year. Because these qualities were generally perceived to be the decisive ones registrars who had not undertaken a psychiatry $\mathrm{SHO}$ post did not report feeling at a disadvantage.

There is always material you can read up. So I feel fine without the training. I mean 
I had to do a bit of reading around medication and how to tailor it, what's your first line and the wash out phases, but that was OK. We have a good support network here. I can always call someone up if I am unsure (13).

None of the registrars suggested that psychiatry SHO posts were indispensable and so it could be that these posts do not constitute a core element of training for GP registrars. It is arguable as to whether resources should be directed to making more community-based psychiatric SHO posts available, or whether time and money are better spent on the registrars when they are in their GP posts. On the one hand, as Dowrick et al. (2000) and Thompson et al. (2000) suggest, confidence is such a crucial aspect of a GP's ability to detect depression that the benefit of psychiatry SHO jobs should not be completely neglected. On the other hand, confidence is likely to increase through many different types of training schemes and therefore focusing resources into primary care-based mental health training would appear sensible. Most important however, is to keep a broad view, investigate the diversity of training experiences available and not to forget the idiosyncrasies attached to each one.

\section{Current support}

The registrars all indicated that they enjoyed their VTS and found it a valuable source of support both socially as well as medically. While mental health and communication skills appeared to be discussed quite frequently during VTS sessions, on the whole registrars spoke more generally about support they received via this programme.

At the end of every session we have a little discussion group where we discuss 'what happened to you in the week?' - almost $80 \%$ of those problems are mental health problems ... There's always a mental health component to it (5).

Registrars also valued the encouragement and support they received and could access within their training practice. They appreciated a close and comfortable relationship with their GP trainers, as well as the expertise of other practice staff, especially in relation to difficult diagnoses, medication and referrals. These personal links were preferred to accessing information from directories, information booklets and the internet.

It is good, there is always someone to call up when you are worried about a patient (2).

I guess you should always have these leaflets somewhere there with you in your bag. But to be honest I prefer just calling someone quickly (3).

It became clear that trainers and in practice colleagues were perceived as potential role models. Many registrars observed individuals' practices and approaches to adopt these into their own way of dealing with patients and handling daily routine. These accounts highlight the influence GP trainer and practice culture could have on registrars' future practice, and thus suggest the potential resource they bear in relation to training provision and support.

I kind of take account of all the different kind of styles I think I can amalgamate together with my personality to be a better kind of doctor. I take little bits, particularly from my trainer (14).

I have a lecture with my trainer at least once a week that I prepare for. And I pick topics that I commonly see so that I get acquainted with how things are done at this practice. Mental health is often an issue because I see it so much. So it is nice to get a firm idea of exactly who needs what kind of approach and treatment, etc. (7).

\section{Conclusion}

\section{Limitations}

Due to the small scale and qualitative approach of this study, generalizations should be made with some caution. GP registrars were interviewed as a particular group of doctors. Their beliefs and issues are likely to differ from those of senior GPs. It was not part of this study to validate registrars' accounts either against their actual behaviours or against their trainers', colleagues' or patients' opinions. Registrars may have found it 
socially and professionally desirable to demonstrate commitment to mental health problems. Also, the fact that the interviews took place in the training practices might have made them less comfortable expressing criticism. Although the interviewees attended different medical schools, they were receiving the same vocational training at the time of the interviews. Specific aspects they valued about this scheme could not be compared and have therefore not been included into this report. Against these limitations, however, the study revealed some crucial aspects about the way new doctors feel about dealing with patients' mental health issues which can explain data from other studies and point to areas of improvement.

We feel this study has shown that these new entrants to general practice were aware of the psychosocial nature of general practice and recognize the prevalence of depression and anxiety. They were both ready and willing to take up the challenge of diagnosing and managing the rising numbers of patients with mental health problems despite their concerns about the costs in both time and their own emotional reserves. They were also broadly confident in their skills to detect even subtle mental health problems and recognize somatization.

However, a diagnosis of depression is not a straightforward action. It is a process that involves negotiation between patient and doctor and a mutual willingness to discuss the pertinent issues. Such a process can span across several consultations. It also involves practical resources such as time, and training issues such as knowledge of and confidence in available treatments. Our findings provide a qualitative explanation to earlier studies which showed that a substantial amount of depression is not diagnosed in the first consultation. They also add substance to those that demonstrate firstly the association between confidence, optimism, communication skills and diagnosing depression, and second, the problems posed by some patients' somatizing attribution styles.

Confidence discussing emotional problems and skilled communication were being acquired through increased experience in general practice, and the highly valued support of the GP trainers and the VTS. However, our findings suggest that optimism in effective management might be held up by poorly refined psychosocial understandings of common mental health problems and a narrow knowledge of community resources, referral options and differences in therapeutic options. This is to be expected from new doctors especially those who begin work in an unfamiliar area. The combination of time pressures and high expectations of their own service delivery can create a difficult context for the management of mental health problems. If not addressed effectively, mental health management can thus be a source of insidious insecurity and permanent frustration, affecting both doctor and patient.

The 1000 new primary care mental health workers and 500 gateway workers which were expected to be employed by 2004 as promised by The NHS plan (Secretary of State for Health, 2000) could provide some of the much needed additional support, especially since GP registrars prefer to learn about primary care from experience and other people rather than leaflets and books. As such the role of trainers, too, cannot be overemphasized. Registrars enter general practice with as little as two weeks' undergraduate experience of the specialty, so role models and personal contacts have important functions at the beginning of their careers. It is crucial for training practices to recognize the impact they have on registrars' attitudes and practices. They must introduce the registrar to the local infrastructure of support networks, therapeutic services, and links with the community mental health teams (CMHT). Furthermore, it should be part of the responsibility of the trainer to discuss the many different roles the registrar will be expected to fulfil and to understand his/her limits. In light of the currently increasing role of GP teaching during medical school and the introduction of postregistration GP SHO jobs, it is likely that future GP registrars will have an easier start with primary care mental health.

\section{Conflict of interest}

One of the authors (HL) is a partner in a practice which takes GP registrars, but has no direct role in training nor any involvement with the VTS.

\section{Acknowledgements}

This research was funded by the South London Networks Bursary Training Fund and received 
ethical approval from Wandsworth Local Research Ethics Committee. We would like to thank all participants for the interviews.

\section{References}

Ashworth, M., Clement, S., Sandhu, J., Farley, N., Ramsay, R. and Davies, T. 2002: Psychiatric referral rates and the influence of on-site mental health workers in general practice. British Journal of General Practice 52, 39-41.

Ayuso-Mateos, J.L., Vazquez-Barquero, J.L., Dorwick, C., Lehtinen, V., Dalgard, O.S., Casey, P., Wilkinsen, C., Lasa, L., Page, H., Dunn, G., Wilkinsen, G. and the Odin Group. 2001: Depressive disorders in Europe: prevalence figures from the Odin study. British Journal of Psychiatry 179, 308-16.

Best, A., Gietzen, T. and Henderson, M. 2002: A patient with somatisation. Practitioner 246, 34-42.

Cape, J. 2000: Patient-related therapeutic relationship and outcome in general practitioner treatment of psychological problems. British Journal of Clinical Psychology 39, $383-95$.

Department of Health. 1995: Living in Britain: results from the 1995 General Household Survey. London: The Stationery Office.

Department of Health. 1999a: Saving lives: our healthier nation. London: The Stationery Office.

Department of Health. 1999b: The National Service Framework for Mental Health. London: Department of Health.

Department of Health. 2001: Make it happen. A guide to delivering mental health promotion. London: Department of Health.

Dowrick, C. and Buchan, I. 1995: Twelve month outcome of depression in general practice. Does detection or disclosure make a difference? British Medical Journal 311, 1274-76.

Dowrick, C., Gask, L., Perry, R., Dixon, C. and Usherwood, T. 2000: Do general practitioners' attitudes towards depression predict their clinical behaviour? Psychological Medicine 30, 413-19.

Gask, L., Usherwood, T., Thompson, H. and Williams, B. 1989: Evaluation of a training package in the assessment and management of depression in primary care. Medical Education 32, 190-98.

Glaser, B.G., and Strauss, A.L. 1967: The discovery of grounded theory: strategies for qualitative research. Chicago: Aldine.

Goldberg, D. and Huxley, P. 1992: Common mental disorders. A bio-social model. London: Routledge.

Goldberg, D. and Williams, P. 1991: A user's guide to the general health questionnaire. Windsor: NFER-Nelson Publishing Company Ltd.

Hickie, I.B. 1999: Primary care psychiatry is not specialist psychiatry in general practice. The Medical Journal of Australia 170, 171-73.
Hodges, B., Inch, C. and Silver, I. 2001: Improving the psychiatric knowledge, skills, and attitudes of primary care physicians, 1950-2000: A review. American Journal of Psychiatry 158, 1579-86.

Kerwick, S., Jones, R., Mann, A. and Goldberg D. 1997: Mental health care training priorities in general practice. British Journal of General Practice 47, 225-27.

Kessler, D., Bennewith, O., Lewis, G. and Sharp, D. 2002: Detection of depression and anxiety in primary care: follow up study. British Medical Journal 325, 1016-17.

Kessler, D., Lloyd, K., Lewis, G. and Gray Periera, D. 1999: Cross sectional study of symptom attribution and recognition of depression and anxiety in primary care. British Medical Journal 318, 436-40.

Mind 1998: Understanding mental illness. www.mind.org.uk Retrieved March 2003.

Ohayon, M.M., Priest, R.G., Guilleminault, C. and Caulet, M. 1999: The prevalence of depressive disorder in Britain. Biological Psychiatry 45, 300-307.

Paykel, E.S. and Priest, R.G. 1992: Recognition and management of depression in general practice: consensus statement. British Medical Journal 305, 1198-202.

Paykel, E.S., Tylee, A., Wright, A., Priest, R.G., Rix, S. and Hart, D. 1997: The Defeat Depression Campaign: psychiatry in the public arena. American Journal of Psychiatry 154 (6 Suppl), 59-64.

Paykel, E.S., Hart, D. and Priest, R.G. 1998: Changes in public attitudes to depression during the Defeat Depression Campaign. British Journal of Psychiatry 173, 519-22.

Payne, F., Harvey, K., Jessopp, L., Plummer, S., Tylee, A. and Gournay, K. 2002: Knowledge, confidence and attitudes towards mental health of nurses working in NHS Direct and the effects of training. Journal of Advanced Nursing 40, 549-59.

Ratcliffe, J., Gask, L., Creed, F. and Lewis, B. 1999: Psychiatric training for family doctors: what do GP registrars want and can a brief course provide this? Medical Education 33, 434-38.

Rix, S., Paykel, E.S., Lelliott, P., Tylee, A., Freeling, P., Gask, L. and Hart, D. 1999: Impact of a national campaign on GP education: an evaluation of the Defeat Depression Campaign. British Journal of General Practice 49, 99-102.

Schilte, A.F., Portegijs, P.J., Blankenstein, A.H., van Der Horst, H.E., Latour, M.B., van Eijk, J.T. and Knottnerus, J.A. 2001: Randomised controlled trial of disclosure of emotionally important events in somatisation in primary care. British Medical Journal 323, 86-91.

Scott, J., Thorne, A. and Horn, P. 2002: Quality improvement report: Effect of a multifaceted approach to detecting and managing depression in primary care. British Medical Journal 325, 951-54.

Secretary of State for Health. 2000: The NHS plan. A plan for investment. A plan for reform. London: The Stationery Office. 
The European Network for Health Promoting Agencies. 2003: www.eurohealthnet.org Retrieved March 2003.

The American Psychiatric Association. 1994: Diagnostic and statistical manual of mental disorders IV. Washington: The American Psychiatric Association.

Thompson, C., Kinmonth, A.L., Stevens, L., Peveler, R.C., Stevens, A., Ostler, K.J., Pickering, R.M., Baker, N.G., Henson, A., Preece, J., Cooper, D. and Campbell, M.J. 2000: Effects of a clinical-practice guideline and practicebased education on detection and outcome of depression in primary care: Hamphire Depression Project randomised controlled trial. Lancet 355, 185-91.
Upton, M.W.M., Evans, M., Goldberg, D.P. and Sharp, D.J. 1999: Evaluation of ICD-10 PHC mental health guidelines in detecting and managing depression within primary care. British Journal of Psychiatry 175, 476-82.

Williams, K. 1998: Self-assessment of clinical competence by general practioner trainees before and after a six month psychiatric placement. British Journal of General Practice 48, 1387-90.

World Health Organization. 1999: The World Health Report. Geneva: World Health Organization. 